\title{
Tumor carcinoide del apéndice cecal: cuando un hallazgo incidental modifica drásticamente el pronóstico y tratamiento del paciente
}

\author{
A Carcinoid Tumor of the Cecal Appendix: When an Incidental Finding \\ Dramatically Modifies a Patient's Prognosis and Treatment
}

María Carolina Díaz Rivera, MD, ${ }^{1}$ Kenny Buitrago-Toro, MD, ${ }^{2}$ Pablo Gonzales, MD. ${ }^{3}$

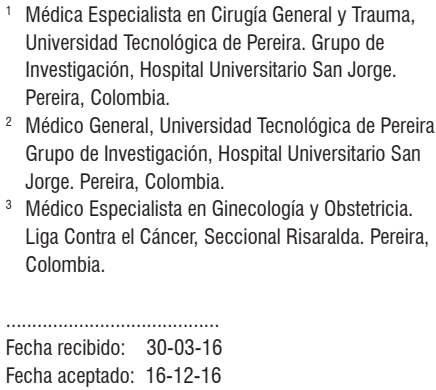

Médica Especialista en Cirugía General y Trauma, Universidad Tecnológica de Pereira. Grupo de Investigación, Hospital Universitario San Jorge. Pereira, Colombia.

2 Médico General, Universidad Tecnológica de Pereira Grupo de Investigación, Hospital Universitario San Jorge. Pereira, Colombia.

3 Médico Especialista en Ginecología y Obstetricia. Liga Contra el Cáncer, Seccional Risaralda. Pereira, Colombia.

Fecha recibido: $\quad 30-03-16$

Fecha aceptado: 16-12-16

\begin{abstract}
Resumen
Introducción: la apendicectomía es un procedimiento ampliamente utilizado en todo el mundo, bien sea como consecuencia de signos y síntomas sugestivos de apendicitis aguda o como resección profiláctica. El estudio de la pieza quirúrgica es frecuente, a pesar de la baja incidencia de hallazgos inusuales en esta. Los tumores del apéndice cecal constituyen el $1 \%$ de todas las neoplasias intestinales, siendo los de tipo carcinoide los más frecuentes en el apéndice cecal. Objetivo: exponer el caso de un tumor carcinoide del apéndice cecal como hallazgo incidental por apendicectomía profiláctica laparoscópica en un procedimiento ginecológico. Caso clínico: paciente de 58 años de edad, con antecedente patológico de miomatosis uterina e histerectomía en 2010, que consulta por cuadro clínico de dos años de evolución de dolor pélvico crónico. Es llevada a liberación de adherencias intraperitoneales que afectan el apéndice cecal, por lo que se realiza apendicetomía, que en el análisis histopatológico identifica tumor carcinoide típico. Discusión: los tumores carcinoides son neoplasias neuroendocrinas que pueden ser identificadas en múltiples localizaciones; sin embargo, el tracto gastrointestinal es el más frecuente. El compromiso de apéndice cecal no es común y suele ser detectado incidentalmente durante apendicectomías profilácticas. La identificación de este tipo de neoplasias en un estudio de rutina modificará drásticamente el manejo del paciente, ya que dependiendo de su tamaño, extensión y localización, requerirá un manejo y seguimiento específico.
\end{abstract}

\section{Palabras clave}

Tumor carcinoide, neoplasia del apéndice, apéndice cecal, apendicectomía.

\section{Abstract}

Introduction: Appendectomies are widely used all over the world when there are signs and symptoms suggestive of acute appendicitis and also for prophylactic resection. Study of the surgical specimen is frequent despite the low incidence of unusual findings. Tumors of the cecal appendix constitute $1 \%$ of all intestinal neoplasms. Among them, carcinoid tumors are the most frequent. Objective: We present a case of a carcinoid tumor of the cecal appendix found incidental to a prophylactic laparoscopic appendectomy during a gynecological procedure. Case report: A 58-year-old patient with a history of uterine myomatosis who had had a hysterectomy in 2010 consulted after two years of chronic pelvic pain. An appendectomy was performed because intraperitoneal adhesions from the surgery had affected the cecal appendix. The histopathological analysis identified a typical carcinoid tumor. Discussion: Carcinoid tumors are neuroendocrine neoplasms that can be found in various locations but which are most common in the gastrointestinal tract. Involvement of the cecal appendix is not common and is usually detected incidental to prophylactic appendectomies. Identification of this type of neoplasia in a routine study drastically modifies patient management because management and follow-up depending on the tumor's size, extent and location.

\section{Key words}

Carcinoid tumor, appendix neoplasia, cecal appendix, appendectomy 


\section{INTRODUCCIÓN}

La resección del apéndice cecal, por vía laparoscópica o abierta, es un procedimiento ampliamente practicado en el mundo, siendo superior a los 300000 casos/año en países como Estados Unidos (1), bien sea como manejo definitivo de una apendicitis aguda o realizado de forma profiláctica al momento de llevar a cabo un procedimiento quirúrgico intraabdominal por otra causa (2).

El estudio histopatológico de la pieza quirúrgica se realiza rutinariamente; sin embargo, la poca frecuencia en la que se encuentran hallazgos inusuales en ella hace que en la literatura aún se cuestione su utilidad (3). En un estudio retrospectivo a 14 años se encontró que de 1466 apendicectomías practicadas, el porcentaje de hallazgos inusuales correspondía al 3,88\%; de estos, los tumores neuroendocrinos correspondían al 0,47\% (4). A pesar de la baja incidencia de hallazgos histopatológicos anormales, el hecho de identificarlos lleva a que el tratamiento y pronóstico cambie tanto, que su uso se hace indispensable (5).

Las neoplasias del apéndice cecal corresponden al 1\% de las neoplasias del tracto gastrointestinal. Pueden ser de diversos tipos, siendo los carcinoides las más comunes, con frecuencias que van desde el $11 \%$ hasta el $50 \%$. Son factores de riesgo la raza negra, el sexo femenino y la edad (pico máximo de incidencia entre la quinta y sexta década de la vida) $(6,7)$.

\section{CASO CLÍNICO}

Paciente de sexo femenino de 58 años de edad, con antecedente patológico de hipertensión arterial y miomatosis uterina, que fue manejada con histerectomía en el 2010 y que consulta en múltiples oportunidades a la unidad de atención primaria por cuadro clínico de dolor tipo sordo en el hemiabdomen inferior, permanente, con episodios de exacerbación, sin desencadenantes ni atenuantes y que no estaba relacionado con ninguna otra sintomatología. Niega síntomas gastrointestinales, ginecológicos o respiratorios, por lo que se da manejo sintomático hasta por dos años.

Ulteriormente es derivada a valoración especializada de ginecología por cuadro de dolor pélvico crónico, razón por la que, basado en el hallazgo ultrasonográfico de adherencias intraperitoneales múltiples, es llevada a cirugía para liberación de estas. Intraoperatoriamente se consulta a la especialidad de cirugía general por amplio compromiso de estructuras intraperitoneales, incluido el apéndice, que es resecado y enviado a estudio histopatológico, realizado de rutina. El procedimiento termina sin complicaciones.

Estando la paciente con recuperación completa, tanto de la cirugía como del cuadro clínico que aquejaba, se recibe el reporte histopatológico de la muestra obtenida durante el procedimiento quirúrgico. Este indica: tumor neuroen- docrino bien diferenciado (carcinoide típico) de $6 \mathrm{~mm}$ de diámetro en la punta apendicular, que compromete la muscular y la serosa, con borde proximal libre de tumor. Por tanto, la paciente es derivada inmediatamente al servicio de oncología para estudios complementarios y manejo.

\section{DISCUSIÓN}

Los tumores carcinoides son neoplasias neuroendocrinas bien diferenciadas que pueden ser halladas en el tracto gastrointestinal (55\%), el tracto respiratorio (30\%) y otros (15\%), como riñones u ovarios. En el tracto gastrointestinal, el intestino delgado corresponde aproximadamente al $45 \%$; el recto, al 20\%; el apéndice cecal, al 16\%; el colon, al $11 \%$; y el estómago, al 7\% (8).

Dependiendo de su extensión y célula progenitora principal, los tumores carcinoides pueden producirgran cantidad de productos neuroendocrinos, tales como insulina, dopamina, gastrina, serotonina, glucagón, péptido intestinal vasoactivo, histamina, somatostatina, entre otros, que actuarán sobre los receptores endógenos y producirán, dependiendo de la cantidad liberada, una sintomatología específica (9).

El enrojecimiento (o flushing) facial asociado con hipotensión, que suele ser episódico, de duración entre 30 segundos y 30 minutos, que puede llegar a afectar el cuello y el tórax superior; la diarrea de tipo secretora; las telangiectasias perinasales o malares; el broncoespasmo; e inclusive, los signos de insuficiencia cardíaca por disfunción valvular conforman el conjunto de síntomas que constituyen el síndrome carcinoide. Sin embargo, su aparición dependerá del tamaño y compromiso metastásico del tumor, y esta es frecuente en neoplasias del intestino delgado, aunque infrecuente en tumores carcinoides del apéndice cecal, que usualmente es asintomático, como ocurrió en nuestra paciente $(9,10)$.

Los tumores del apéndice cecal pueden afectar hasta en un $10 \%$ la base de este, lo que lleva, a su vez, dependiendo de su tamaño y extensión, a obstrucción de la luz intestinal y del flujo linfático y venoso, a edema e isquemia, que producen una respuesta inflamatoria sistémica y la sintomatología clásica de apendicitis aguda. No obstante, la gran mayoría de casos en los cuales se detecta esta neoplasia suelen ser hallazgos ocasionales secundario a apendicectomías profilácticas en procedimientos quirúrgicos de otra índole, como es el caso de la paciente presentada $(6,11)$.

El estudio histopatológico es de vital importancia para la detección de las células tumorales, su tipo histológico, las márgenes de resección libres de células cancerosas, el compromiso basal o distal del apéndice y el tamaño del tumor, incluida su invasión de las diferentes capas de la mucosa, características que serán útiles al momento de determinar la terapia definitiva, que se basa más en el consenso de expertos que en los estudios de gran poder estadístico. En 
aquellos tumores con compromiso distal $<1 \mathrm{~cm}$, la apendicectomía usualmente es curativa. Por su parte, para los $>2 \mathrm{~cm}$, la hemicolectomía estará indicada para erradicar el compromiso metastásico colónico y ganglionar. Entre 1 y $2 \mathrm{~cm}$, el tratamiento se diferencia en dos grupos: aquellos sin compromiso de la base, en los que se prefiere la apendicectomía como único tratamiento, o aquellos que reportan en el estudio histopatológico bordes positivos, angioinvaginación, tumor mixto o afección del mesoapéndice, en los cuales el tratamiento es más agresivo y se opta por la hemicolectomía $(6,10)$. Dependiendo del tamaño del tumor, este manejo se utiliza basado en la premisa y en los resultados de estudios que demuestran que el compromiso metastásico es casi nulo para tumores menores de $1 \mathrm{~cm}$, del $7,5 \%$ para los que miden entre 1 y $2 \mathrm{~cm}$, y hasta del $33 \%$ para los mayores de $2 \mathrm{~cm}$ (12).

El pronóstico de los pacientes con tumores del apéndice cecal también se verá afectado tanto por su tamaño como por el compromiso metastásico que tenga la neoplasia. Tumores menores de $2 \mathrm{~cm}$, sin compromiso a distancia, tendrán tasas de sobrevida a los 5 años cercanas al 100\%; sin embargo, los tumores entre 1 y $2 \mathrm{~cm}$, con metástasis ganglionar o mayores de $2 \mathrm{~cm}$, la sobrevida descenderá al 78\%, en el mismo rango de tiempo. Cuando el compromiso metastásico es orgánico, generalmente hepático en este tipo de tumores, independientemente del tamaño, caerá al 32\% $(13,14)$.

El seguimiento de esta patología se realiza en función del tamaño detectado. Los tumores con tamaño $<2 \mathrm{~cm}$, en los que la apendicectomía es considerada como el manejo definitivo, no requieren seguimiento. En las neoplasias entre 1 y $2 \mathrm{~cm}$ o $>2 \mathrm{~cm}$ hemicolectomizadas, se sugiere el seguimiento imagenológico con tomografía axial computarizada (TAC) abdominal con doble contraste, octreoscan o serológica, que identifica marcadores como la cromogranina y que se llevan a cabo anualmente para determinar el compromiso hepático metastásico o la sintomatología sugestiva de síndrome carcinoide. Para aquellos en los cuales se demuestre metástasis a distancia, el seguimiento está indicado cada 6 meses (15).

Ulteriormente la paciente fue derivada a la unidad de oncología de otra institución y está actualmente en seguimientos periódicos. Los estudios subsecuentes han sido negativos para malignidad. Queremos recalcar la importancia del estudio histopatológico de las piezas quirúrgicas, a pesar del bajo porcentaje de hallazgos inusuales, dados los diferentes esquemas de tratamiento que deben emplearse dependiendo de este resultado.

\section{REFERENCIAS}

1. Flum DR. Acute appendicitis - Appendectomy or the “antibiotics first” strategy. N Eng J Med. 2015;372:1937-43.

2. Occhionorelli S, Stano R, Targa S, et al. Prophylactic appendectomy during laparoscopic surgery for other conditions. Case Rep Med. 2014;2014:292864.

3. Guraya SY. Do we still need to perform routine histological examination of appendectomy specimens? J Clin Diag Res. 2015;9:PL01-PL.

4. Yabanoglu H, Caliskan $\mathrm{K}$, Aytac $\mathrm{HO}$, et al. Unusual findings in appendectomy specimens of adults: retrospective analyses of 1466 patients and a review of literature. Iranian Red Crescent Med J. 2014;16(2):e12931.

5. Omiyale AO, Adjepong S. Histopathological correlations of appendectomies: a clinical audit of a single center. Ann Transl Med. 2015;3(9):119.

6. Shankar S, Ledakis P, El Halabi H, et al. Neoplasms of the appendix: current treatment guidelines. Hematol Oncol Clin North Am. 2012;26(6):1261-90.

7. Hemminki K, Li X. Incidence trends and risk factors of carcinoid tumors. Cancer. 2001;92:2204-10.

8. Maggard MA, O'Connell JB, Ko CY. Updated population-based review of carcinoid tumors. Ann Surg. 2004;240(1):117-22.

9. Kunz PL. Carcinoid and neuroendocrine tumors: building on success. J Clin Oncol. 2015;33(16):1855-63.

10. Bolanowski M, Bednarczuk T, Bobek-Billewicz B, et al. Neuroendocrine neoplasms of the small intestine and the appendix - management guidelines (recommended by the Polish Network of Neuroendocrine Tumours). Endokrynol Pol. 2013;64(6):480-93.

11. Gu Y, Wang N, Xu H. Carcinoid tumor of the appendix: a case report. Oncol Lett. 2015;9(5):2401-3.

12. Rorstad O. Prognostic indicators for carcinoid neuroendocrine tumors of the gastrointestinal tract. J Surg Oncol. 2005;89:151-60.

13. Turaga KK, Pappas SG, Gamblin TC. Importance of histologic subtype in the staging of appendiceal tumors. Ann Surg Oncol. 2012;19:1379-85.

14. Landry CS, Woodall C, Scoggins CR, et al. Analysis of 900 appendiceal carcinoid tumors for a proposed predictive staging system. Arch Surg. 2008;143:664-70.

15. Boudreaux JP, Klimstra DS, Hassan MM, et al. The NANETS consensus guideline for the diagnosis and management of neuroendocrine tumors: well-differentiated neuroendocrine tumors of the jejunum, ileum, appendix, and cecum. Pancreas. 2010;39:753-66. 